\title{
Tunable one-dimensional microwave emissions from cyclic-transition three-level artificial atoms
}

\author{
W. Z. Jia, ${ }^{1}$ L. F. Wei, ${ }^{1,2}$ and Z. D. Wang ${ }^{3}$ \\ ${ }^{1}$ Quantum Optoelectronics Laboratory, School of Physics and Technology, Southwest Jiaotong University, Chengdu 610031, China \\ ${ }^{2}$ State Key Laboratory of Optoelectronic Materials and Technologies, School of Physics and Engineering, \\ Sun Yat-Sen University, Guangzhou 510275, China \\ ${ }^{3}$ Department of Physics and Center of Theoretical and Computational Physics, The University of Hong Kong, \\ Pokfulam Road, Hong Kong, China \\ (Received 29 October 2010; published 16 February 2011)
}

\begin{abstract}
By strongly driving a cyclic-transition three-level artificial atom, demonstrated by such as a flux-based superconducting circuit, we show that coherent microwave signals can be excited along a coupled one-dimensional transmission line. Typically, the intensity of the generated microwave is tunable via properly adjusting the Rabi frequencies of the applied strong-driving fields or introducing a probe field with the same frequency. In practice, the system proposed here could work as an on-chip quantum device with controllable atom-photon interaction to implement a total-reflecting mirror or switch for the propagating probe field.
\end{abstract}

DOI: 10.1103/PhysRevA.83.023811

PACS number(s): 42.50.Pq, 42.50.Gy, 85.25.-j, 42.50.Hz

\section{INTRODUCTION}

Superconducting quantum circuits (SQCs) can be regarded as artificial atoms (AAs) with quantized energy levels [1]. Quantum mechanical behaviors in these AAs, such as spectroscopy [2-4], Rabi oscillations [5,6], and so forth, have already been demonstrated experimentally. Additionally, strong coupling between an AA and a single-mode microwave field in a high- $Q$ resonator can realize a macroscopic analog of the cavity quantum electrodynamics (QED), known as circuit QED $[7,8]$. However, when such an atom interacts with waves propagating freely along an open 1D transmission line (TL), the situation differs qualitatively. In this case, the system should be described by the continuous electromagnetic fields being scattered by a pointlike AA [9-12]. Differing from the usual 3D scattering process, here the problem of spatial-mode mismatch between the incident and scattered waves can be effectively overcome as these waves are confined in the 1D space $[9,10]$. This also provides a way to demonstrate the strong interactions between the 1D microwave fields and an AA based on flux-biased superconducting circuit [10-12]. Specifically, by virtue of the tunability, controllability, and strong atom-photon coupling, these on-chip macroscopic quantum devices could be utilized to reproduce certain typical quantum optical phenomena, e.g., resonance fluorescence [10], electromagnetically induced transparency (EIT) [11], and ultimate on-chip amplifier [12], and so on.

In this article, we discuss the feasibility of generating microwave with specific frequency along an open 1D TL by manipulating the strong-field-induced coherence in a coupled three-level $\Delta$-type AA $[1,13,14]$. The produced microwave emission can be controlled by introducing another weak probe field with the same frequency. Our results display some special 1D quantum optical phenomena in the microwave domain and provide the potential applications in on-chip photonic quantum information processings with SQCs.

\section{TUNABLE MICROWAVE EMISSIONS FROM A $\triangle$-TYPE ARTIFICIAL ATOM}

We consider a system schematized in Fig. 1, where a superconducting loop interrupted by four Josephson junctions couples to an open 1D TL through the loop-line mutual inductance $M$. The loop can be looked on as a multilevel AA, whose energy levels and transition elements can be tuned by adjusting the external magnetic flux $\Phi$. At some working points [12], the desirable three-level $\Delta$-type transition configurations can be realized $[13,14]$. Here we assume $|i\rangle(i=1,2,3)$ and $\omega_{i}$ are the three selected levels and their eigenfrequenies of the $\Delta$-type AA. Three coherent microwave driving fields with the actual currents $\frac{1}{2} I_{i j} e^{i k_{i j} x-v_{i j} t}+$ c.c. $(i, j=1,2,3, i>j)$, propagating along the open 1D TL with the wave vectors $k_{i j}$ and frequencies $v_{i j}$, are applied to couple the three possible transition-channels of the pointlike AA located at $x=0$. The Hamiltonian of this system can be written as

$$
\hat{H}=\hbar \sum_{i=1}^{3} \omega_{i} \hat{\sigma}_{i i}-\frac{\hbar}{2} \sum_{i>j=1}^{3}\left(\Omega_{i j} e^{-i v_{i j} t} \hat{\sigma}_{i j}+\text { H.c. }\right) \text {. }
$$

Here $\hat{\sigma}_{i j}=|i\rangle\langle j|$ are the atomic projection or transition operators, and $\Omega_{i j}=\phi_{i j} I_{i j} / \hbar$ are the Rabi frequencies. The dipole moment matrix elements can be written as $\phi_{i j}=t_{i j} M I_{P}$, where $t_{i j}\left(=t_{j i}\right)$ are the dimensionless matrix elements, $M$ is the line-atom mutual inductance, and $I_{P}$ is the amplitude of the persistent current in the loop.

In the following, we will show that two strong-driving fields $\Omega_{31}$ and $\Omega_{32}$ can induce an effective magnetic flux $\phi$ with oscillating frequency $v_{21}=v_{31}-v_{32}$ in the AA loop. And consequently, the AA as a pointlike source, will emit microwaves with frequency $v_{21}$ in the TL. Specifically, we assume $\Omega_{31}$ is resonantly applied between the levels $|1\rangle$ and $|3\rangle$, and $\Omega_{32}$ applied between the levels $|2\rangle$ and $|3\rangle$ with a detuning $\Delta_{32}=v_{32}-\omega_{3}+\omega_{2}$, as shown in Fig. 2(a). In the interaction picture, the Hamiltonian of this driven three-level system reads

$$
\hat{H}_{I}=\hbar \Delta_{32} \hat{\sigma}_{22}-\frac{\hbar}{2}\left(\Omega_{31} \hat{\sigma}_{31}+\Omega_{32} \hat{\sigma}_{32}+\text { H.c. }\right)
$$

and its corresponding dynamics can be described by the master equation

$$
\dot{\rho}=-\frac{i}{\hbar}\left[\hat{H}_{I}, \rho\right]+L[\rho],
$$




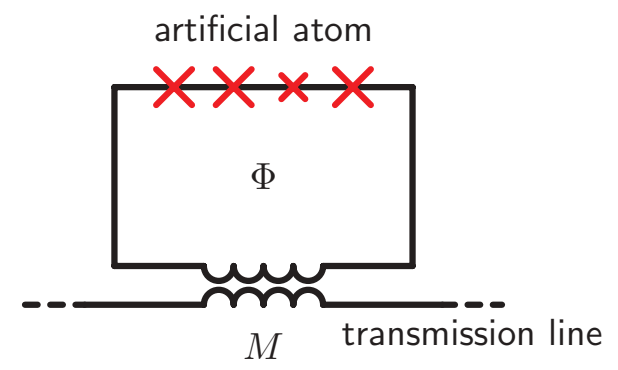

FIG. 1. (Color online) Circuit diagram [10] of an artificial atom (generated by four-junction flux qubit geometry) inductively coupled to a $1 \mathrm{D}$ long transmission line.

with $\rho=\sum_{i j} \rho_{i j} \hat{\sigma}_{i j}$ being the density matrix. The Lindblad term is defined by

$$
\begin{aligned}
L[\rho]= & \Gamma_{31} \rho_{33}\left(\hat{\sigma}_{11}-\hat{\sigma}_{33}\right)+\Gamma_{32} \rho_{33}\left(\hat{\sigma}_{22}-\hat{\sigma}_{33}\right) \\
& +\Gamma_{21} \rho_{22}\left(\hat{\sigma}_{11}-\hat{\sigma}_{22}\right)-\sum_{i \neq j} \gamma_{i j} \rho_{i j} \hat{\sigma}_{i j} .
\end{aligned}
$$

Here $\Gamma_{i j}(i>j)$ are the relaxation rates between the levels $|i\rangle$ and $|j\rangle, \gamma_{i j}=\gamma_{j i}$ are the damping rates of the off-diagonal terms (specifically, $\gamma_{12}=\Gamma_{21} / 2+\Gamma_{12}^{\varphi}, \gamma_{13}=$ $\left(\Gamma_{32}+\Gamma_{31}\right) / 2+\Gamma_{13}^{\varphi}, \gamma_{23}=\left(\Gamma_{32}+\Gamma_{31}+\Gamma_{21}\right) / 2+\Gamma_{23}^{\varphi}$, with $\Gamma_{i j}^{\varphi}$ being the pure dephasings).

Under the usual steady-state condition, the density matrix element $\rho_{21}$ (which describes the coherence between $|1\rangle$ and $|2\rangle)$ can be written as

$$
\rho_{21}=\frac{\Omega_{31} \Omega_{32}^{*}\left[\lambda_{23}\left(\rho_{33}-\rho_{11}\right)+\gamma_{13}\left(\rho_{33}-\rho_{22}\right)\right]}{\left|\Omega_{31}\right|^{2} \gamma_{13}+\lambda_{23}\left(\left|\Omega_{32}\right|^{2}+4 \gamma_{13} \lambda_{21}\right)}
$$

with $\lambda_{21}=\gamma_{12}-i \Delta, \lambda_{23}=\gamma_{23}-i \Delta$. Here $\Delta=-\Delta_{32}$ can be defined as the detuning of the induced wave. Based on the experimental detections [12], the pure dephasing rates in $\gamma_{13}$ and $\gamma_{23}$ can be neglected as they are expected to be weaker than that in $\gamma_{12}$, and additionally $\Gamma_{32} \gg \Gamma_{21}, \Gamma_{31}$ is satisfied. (a)

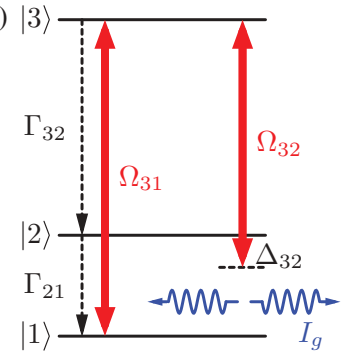

(b) $|3\rangle$

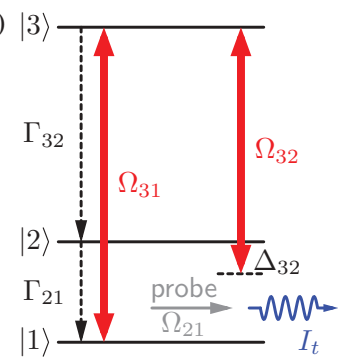

FIG. 2. (Color online) Schematics of the driven three-level artificial atom: (a) Strong fields with Rabi frequencies $\Omega_{31}$ and $\Omega_{32}$ are applied to produce microwave emission between the levels $|1\rangle$ and $|2\rangle$. (b) A weak probe field with Rabi frequency $\Omega_{21}$ is additionally applied to control the microwave emission.
Thus we have $\gamma_{13} \approx \Gamma_{32} / 2, \gamma_{23} \approx \Gamma_{32} / 2$. As a consequence, at resonant point $\Delta=0$, the steady populations read

$$
\left\{\begin{array}{l}
\rho_{11}=\frac{1}{A}\left(2 \gamma_{12}+\Gamma_{32}\right)\left|\Omega_{32}\right|^{2}, \\
\rho_{22}=\frac{1}{A}\left[2 \gamma_{12}\left|\Omega_{32}\right|^{2}+\Gamma_{32}\left(\left|\Omega_{31}\right|^{2}+2 \gamma_{12} \Gamma_{32}\right)\right], \\
\rho_{33}=\frac{1}{A}\left(2 \gamma_{12}\left|\Omega_{32}\right|^{2}\right),
\end{array}\right.
$$

where

$$
A=\left(6 \gamma_{12}+\Gamma_{32}\right)\left|\Omega_{32}\right|^{2}+\Gamma_{32}\left(\left|\Omega_{31}\right|^{2}+2 \gamma_{12} \Gamma_{32}\right) .
$$

Thus $\rho_{21}$ (at resonant point $\Delta=0$ ) can be simplified as

$$
\rho_{21}=-\frac{1}{A}\left(\Gamma_{32} \Omega_{31} \Omega_{32}^{*}\right) .
$$

This implies that an additional magnetic flux threading the AA loop

$$
\phi(t)=\operatorname{Tr}[\hat{\phi}(t) \rho]=\phi_{12} \rho_{21} e^{-i \nu_{21} t}+\text { c.c. }
$$

can be induced, where $\hat{\phi}(t)=\phi_{12} \hat{\sigma}_{12} e^{-i v_{21} t}+$ H.c. is the related atomic dipole-moment operator. Note that this flux is originated from the nonzero dipole-transition elements $\phi_{i j}$ between any pair of levels, which can only be realized in the present $\Delta$-type AA with the broken parity-symmetry.

Certainly, this induced oscillating flux in the AA loop could produce mutually a microwave current $I_{g}(x, t)$ along the TL, which satisfies the relevant $1 \mathrm{D}$ wave equation:

$$
\left(\partial_{x x}-v^{-2} \partial_{t t}\right) I_{g}(x, t)=c \delta(x) \partial_{t t} \phi(t),
$$

where $v=1 / \sqrt{l c}$ is the phase velocity ( $l$ and $c$ are inductance and capacitance per unit length, respectively). The above equation describes a pointlike AA located at $x=0$ with oscillating flux $\phi(t)$ emitting microwave $I_{g}(x, t)$ in the 1D TL. With the relation $\Gamma_{i j}=\left(\hbar \omega_{i j} \phi_{i j}^{2}\right) /\left(\hbar^{2} Z\right)$ (with $Z=\sqrt{l / c}$ being the line impedance) [10], the solution of Eq. (9) can be expressed as $I_{g}(x, t)=\frac{1}{2} I_{g} e^{i k_{21}|x|-i v_{21} t}+$ c.c. (representing the induced waves propagating in both directions) with complex amplitude $I_{g}=i J \rho_{21}$ and $J=\sqrt{\hbar \omega_{21} \Gamma_{21} / Z}$. The dispersion relation is $\nu_{21}=v k_{21}$. Thus the intensity of the induced microwave current reads: $\left|I_{g}\right|=J\left|\rho_{21}\right|$. Specifically, at the resonant point, $\left|I_{g}\right|$ is determined by

$$
\left|I_{g}\right|=\frac{1}{A}\left(J \Gamma_{32}\left|\Omega_{31}\right|\left|\Omega_{32}\right|\right)
$$

and could be controlled via adjusting the values of the applied Rabi frequencies. For example, if $\left|\Omega_{31}\right|$ and $\left|\Omega_{32}\right|$ are modulated synchronously, i.e., $\left|\Omega_{31}\right|=\left|\Omega_{32}\right|=\Omega$, then $\left|I_{g}\right|$ is monotonically increasing with $\Omega$, approaching $J \Gamma_{32} /\left(6 \gamma_{12}+\right.$ $2 \Gamma_{32}$ ) for sufficiently large $\Omega$. Typically, the upper limit of the produced microwave current $\left|I_{g}\right|_{\max }=J / 2$ is achieved for $\gamma_{12} \ll \Gamma_{32}$.

The above microwave emission could be further controlled by applying a probe field $I_{21}(x, t)=\frac{1}{2} I_{21} e^{i k_{21} x-i \nu_{21} t}+$ c.c. to couple the levels $|1\rangle$ and $|2\rangle$, as shown in Fig. 2(b). At the resonant point and under the weak probe approximation, the according steady-state coherence term is

$$
\rho_{21}^{\prime}=-\frac{1}{A}\left(\Gamma_{32} \Omega_{31} \Omega_{32}^{*}\right)+\Omega_{21} B
$$


with

$$
B=\frac{i \Gamma_{32}\left[\Gamma_{32}\left(\left|\Omega_{32}\right|^{2}-\left|\Omega_{31}\right|^{2}\right)+2 \gamma_{12}\left(\left|\Omega_{32}\right|^{2}-\Gamma_{32}^{2}\right)\right]}{A\left(\left|\Omega_{31}\right|^{2}+\left|\Omega_{32}\right|^{2}+2 \gamma_{12} \Gamma_{32}\right)} .
$$

Clearly, the net microwave propagating along the 1D TL reads $I_{t}(x, t)=\frac{1}{2}\left(I_{21} e^{i k_{21} x}+i J \rho_{21}^{\prime} e^{i k_{21}|x|}\right) e^{-i \nu_{21} t}+$ c.c.. And the amplitude of resulting current at $x>0$ can be expressed as $I_{t}=I_{21}+i J \rho_{21}^{\prime}$, namely a superposition of the incident probe and the wave mainly induced by the strong driving fields. Utilizing the expression for $\Omega_{i j}$ and $\Gamma_{i j}$, the intensity of the total microwave current $\left|I_{t}\right|$ can be written as $\left|I_{t}\right|=$ $J\left|\Omega_{21} / \Gamma_{21}+i \rho_{21}^{\prime}\right|$.

For strong driving fields, the second term in Eq. (11) is much smaller than the first term in Eq. (11) and $\Omega_{21} / \Gamma_{21}$ [15] and can then be neglected when calculating $\left|I_{t}\right|$. Note that this approximation is optimal if choosing $\left|\Omega_{31}\right|=\left|\Omega_{32}\right|=$ $\Gamma_{32}$, resulting in vanishing of the second term in Eq. (11). Consequently, at resonant point, $\left|I_{t}\right|$ can be rewritten as

$$
\left|I_{t}\right|=\frac{J\left|\Omega_{21}\right|}{\Gamma_{21}}\left(1+\alpha^{2}-2 \alpha \sin \Theta\right)^{\frac{1}{2}},
$$

with $\alpha=\Gamma_{21} \Gamma_{32}\left|\Omega_{31}\right|\left|\Omega_{32}\right| /\left(A\left|\Omega_{21}\right|\right)$ and $\Theta=\theta_{21}+\theta_{32}-$ $\theta_{31}$ (where $\theta_{i j}$ is the phase factor of $\Omega_{i j}$ ).

The above calculations indicate that (i) for the fixed complex Rabi frequencies $\Omega_{31}$ and $\Omega_{32},\left|I_{t}\right|$ can still be modulated by changing the complex Rabi frequency of the probe $\Omega_{21}=\left|\Omega_{21}\right| e^{i \theta_{21}}$. Particularly, when $\Theta=\pi / 2, \alpha=1$, the induced microwave current at $x>0$ is totally switched off (i.e., $\left|I_{t}\right|=0$ ). Certainly, in this case the induced microwave current at $x<0$ remains unchanged and thus the relevant microwave emission from the AA is unidirectional. (ii) Alternatively, if the probe $\Omega_{21}$ is treated as a signal, the above strong-field-driven AA can be regarded as a total reflection mirror for the probe field. Originally, in the absence of the strong-driving fields, a weak probe field propagating along the 1D TL can be partially absorbed (reflected) by resonantly interacting with the AA. The reflection amplitude could be expressed as $\Gamma_{21} /\left(2 \gamma_{12}\right)$, if the atom-line coupling efficiency is unit [10]. Therefore, only when the pure dephasing $\Gamma_{12}^{\varphi}\left(=\gamma_{12}-\Gamma_{21} / 2\right)$ is negligible can the AA work as the desirable reflection mirror. Our calculations show that this shortcoming could be overcome by appropriately applying the two strong-driving fields $\Omega_{31}$ and $\Omega_{32}$ satisfying $\Theta=$ $\pi / 2 ; \alpha=1$. Under this condition, despite remarkable pure dephasing [note that $\alpha$ is a function of $A\left(\gamma_{12}\right)$ ], the incident probe field can be completely canceled out because of its destructive interference with the strong-field-induced wave. Consequently, an interesting strong-field-induced absorption (reflection) phenomenon can be realized.

\section{EXPERIMENTAL FEASIBILITY}

Immediately, the phenomena predicted above could be verified with the recent experimental device [10-12], i.e., an open 1D TL couples to an AA based on superconducting flux qubit geometry. For the four-junction flux qubit proposed there, by tuning the flux bias $\delta \Phi=\Phi-\Phi_{0} / 2$ (with $\Phi_{0}$ being the flux quantum) slightly apart from the point $\delta \Phi=0$, a cyclic-transition structure can be realized with the lowest three levels. Typically, if the flux bias is set as
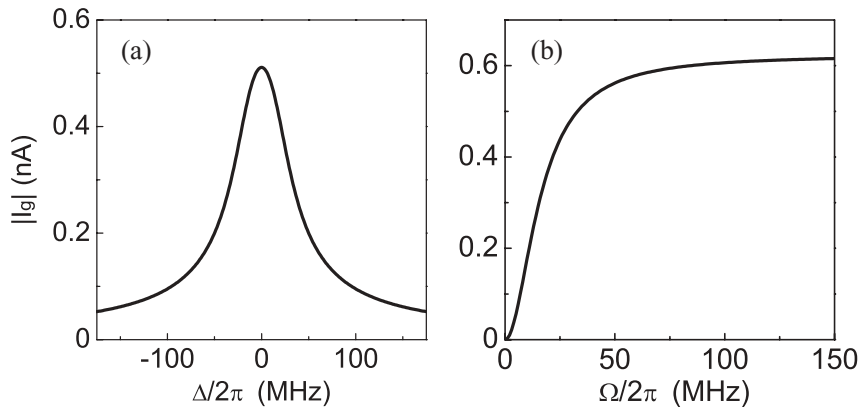

FIG. 3. (a) The microwave emission spectrum induced by strong driving fields $\Omega_{31}$ and $\Omega_{32}$. (b) The intensity of produced microwave current as a function of $\Omega\left(\left|\Omega_{31}\right|=\left|\Omega_{32}\right|=\Omega\right)$ at $\Delta=0$.

$\delta \Phi / \Phi_{0}=3.5 \times 10^{-3}$, the corresponding experimental parameters of AA are $\Gamma_{32} / 2 \pi=35 \mathrm{MHz}, \Gamma_{21} / 2 \pi=11 \mathrm{MHz}$, $\gamma_{12} / 2 \pi=18 \mathrm{MHz}, \omega_{32} / 2 \pi=24.15 \mathrm{GHz}$, and $\omega_{21} / 2 \pi=$ $10.96 \mathrm{GHz}$ [12]. Also, the characteristic impedance of the coplanar TL is $Z \approx 50 \Omega$ [11]. Furthermore, we assume that the Rabi frequencies of the applied two strong-driving fields could be set as $\left|\Omega_{31}\right| / 2 \pi=\left|\Omega_{32}\right| / 2 \pi=35 \mathrm{MHz}$. With these parameters, numerical simulations are carried out, and the results are presented in Figs. 3-5.

We plot the induced microwave emission spectrum in Fig. 3(a). It is seen that, around the resonant point $\Delta=0$, an obvious emission peak appears with maximal amplitude $\left|I_{g}\right| \approx 0.51 \mathrm{nA}$, which is in good agreement with the above theoretical estimation utilizing Eq. (10). In addition, Fig. 3(b) shows the influence of driving fields on $\left|I_{g}\right|$ (here, $\left|\Omega_{31}\right|=$ $\left|\Omega_{32}\right|=\Omega$ ). We can see that a remarkable microwave current can be obtained when the driving fields are comparable with $\Gamma_{32}$. It reaches $0.62 \mathrm{nA}$, if the control fields are strong enough. This is in good accordance with the theoretical prediction $\left[J \Gamma_{32} /\left(6 \gamma_{12}+2 \Gamma_{32}\right)\right]$ given above. To attain a more remarkable induced current, one can enhance either $\left|\rho_{21}\right|$ or $J$. On one hand, a smaller decoherence rate $\gamma_{12}$ may lead to a larger $\left|\rho_{21}\right|$. Note that in the limit of $\gamma_{12} \ll \Gamma_{32}$, the upper limit of induced current is $J / 2=1.58 \mathrm{nA}$. On the other hand, as $J=\sqrt{\hbar \omega_{21} \Gamma_{21} / Z}$, one can enlarge $J$ by choosing the sample with the smaller line impedance $Z$ and larger loop-line mutual inductance $M$.
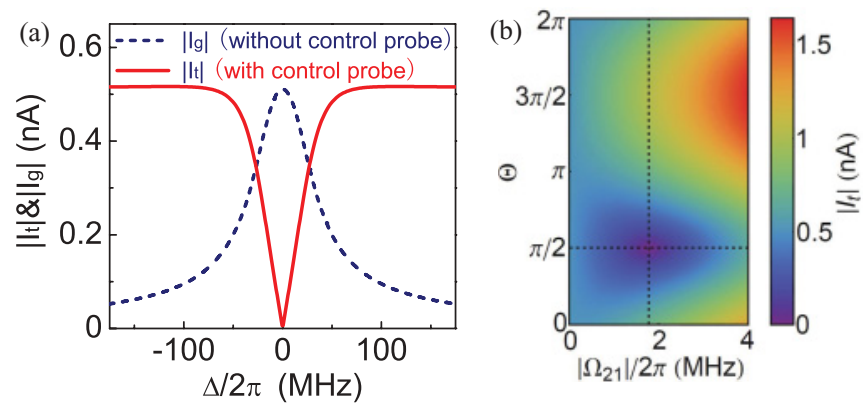

FIG. 4. (Color online) Switch-off the produced microwave emission by a probe field. (a) Induced microwave emission spectrum without control probe (dashed line), which can be switched off at resonant point by a control probe $\Omega_{21}$ (solid line). (b) The intensity plot displays the optimal working point for the probe to switch off the microwave emission: $\Theta=\pi / 2,\left|\Omega_{21}\right| / 2 \pi=1.78 \mathrm{MHz}$. 

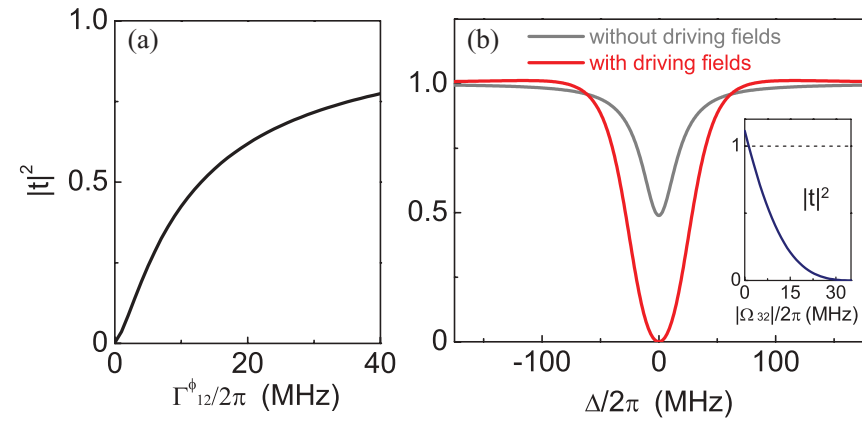

FIG. 5. (Color online) (a) When no driving fields applied, the influence of pure dephasing $\Gamma_{12}^{\varphi}$ on the power transmission coefficient $|t|^{2}$ for a resonantly incident weak probe with $\left|\Omega_{21}\right| / 2 \pi=1.78 \mathrm{MHz}$. (b) The power transmission coefficient $|t|^{2}$ as a function of probe detuning, displaying partial absorption when no driving fields applied and strong-field-induced absorption, respectively. The inset shows single AA quantum switch for resonant probe signal, realized by fixing $\left|\Omega_{31}\right|$ and modulating $\left|\Omega_{32}\right|$.

Figure 4(a) shows that when a probe field with $\Theta=$ $\pi / 2,\left|\Omega_{21}\right| / 2 \pi=1.78 \mathrm{MHz}$ (satisfying $\alpha=1$ ) is applied, the original induced microwave emission peak [dashed line, repeating the spectrum in Fig. 3(a)], will be changed into a dip with zero emission at resonant point (solid line). This indicates that the total suppression for the emission current at $x>0$, which is in accordance with the above theoretical estimation. Figure 4(b) displays $\left|I_{t}\right|$ as a function of $\left|\Omega_{21}\right|$ and $\Theta$ (the detuning is fixed at $\Delta=0$ ). This verifies that the probe parameters used in Fig. 4(a) are optimal for implementing the desirable switch-off operation.

By setting $\Omega_{31}=\Omega_{32}=0$, the power transmission coefficient $|t|^{2}\left(t=I_{t} / I_{21}\right)$ as a function of pure dephasing $\Gamma_{12}^{\varphi}$ (other atom parameters are the same as previous discussion), for a resonantly incident weak probe with $\left|\Omega_{21}\right| / 2 \pi=$ $1.78 \mathrm{MHz}$, is shown in Fig. 5(a). One can see that only in the ideal case of $\Gamma_{12}^{\varphi}=0$ can the atom be regarded as a total reflection mirror with $|t|^{2}=0$. However, when the pure dephasing is not negligible, only partial power can be reflected by the atom. Typically, for a probe with $\left|\Omega_{21}\right| / 2 \pi=1.78$ $\mathrm{MHz}$ interacting with an AA with pure dephasing, $\Gamma_{12}^{\varphi} / 2 \pi=$ 12.5 MHz (from the AA parameters in Ref. [12]), at the resonant point the according power transmission coefficient $|t|^{2}$ is only about 0.49 [gray line in Fig. 5(b)]. When the two strong fields with $\left|\Omega_{31}\right| / 2 \pi=\left|\Omega_{32}\right| / 2 \pi=35 \mathrm{MHz}$ (satisfying $\alpha=1), \Theta=\pi / 2$ are applied, the power extinction for the same resonantly incident probe is reaching the ideal case of $100 \%$ [red line in Fig. 5(b)], displaying the phenomenon of strong-field-induced absorption (single atom total reflection mirror). Moreover, the inset in Fig. 5(b) shows by fixing other parameters and decreasing $\left|\Omega_{32}\right|$ from $2 \pi \times 35 \mathrm{MHz}$ to about $2 \pi \times 1.3 \mathrm{MHz}$, the resonant probe signal can be tuned from completely reflection to totally transmission, demonstrating a perfect single AA switch for propagating probe. Also, the inset verifies when $\left|\Omega_{32}\right|=0$, the AA will work as an on-chip amplifier [12].

\section{CONCLUSIONS AND DISCUSSIONS}

In summary, we have proposed an approach to realize strong-field-induced microwave emission by single AA coupled to 1D open space of a TL. Moreover, we have shown that this kind of induced microwave emission is controllable and tunable by another weak probe. Although various quantum optical effects based on multilevel structure of superconducting AAs have been demonstrated in a series of recent experiments [11,16-18], our results show certain addional effects in the 1D quantum optics at microwave regime. On the other hand, the quantum devices with these effects may have interesting applications in photonic quantum information processing utilizing SQCs. Substantially, the generation of induced microwave emission is a frequency conversion process. This indicates that the proposed device may be used to connect superconducting quantum devices operating at different frequencies in the future SQCs. Finally, the device can also be used as tunable coherent microwave source or total reflection mirror (single AA quantum switch) controlling the propagation of $1 \mathrm{D}$ probe field.

\section{ACKNOWLEDGMENTS}

The project was supported in part by National Natural Science Foundation of China under Grants No. 10874142 and No. 90921010, and the National Fundamental Research Program of China through Grant No. 2010CB923104.
[1] J. Clark, A. N. Cleland, M. H. Devoret, D. Esteve, and J. M. Martinis, Science 239, 992 (1988)

[2] J. R. Friedman, V. Patel, W. Chen, S. K. Tolpygo, and J. E. Lukens, Nature (London) 406, 43 (2000).

[3] C. H. van der Wal, A. C. J. ter Haar, F. K. Wilhelm, R. N. Schouten, C. J. P. M. Harmans, T. P. Orlando, S. Lloyd, and J. E. Mooij, Science 290, 773 (2000).

[4] A. J. Berkley, H. Xu, R. C. Ramos, M. A. Gubrud, F. W. Strauch, P. R. Johnson, J. R. Anderson, A. J. Dragt, C. J. Lobb, and F. C. Wellstood, Science 300, 1548 (2003).

[5] D. Vion, A. Aassime, A. Cottet, P. Joyez, H. Pothier, C. Urbina, D. Esteve, and M. H. Devoret, Science 296, 886 (2002).

[6] Chiorescu, Y. Nakamura, C. J. P. M. Harmans, and J. E. Mooij, Science 299, 1869 (2003).
[7] A. Wallraff, D. I. Schuster, A. Blais, L. Frunzio, R. S. Huang, J. Majer, S. Kumar, S. M. Girvin, and R. J. Schoelkopf, Nature (London) 431, 162 (2004).

[8] A. Blais, R. S. Huang, A. Wallraff, and S. M. Girvin, and R. J. Schoelkopf, Phys. Rev. A 69, 062320 (2004).

[9] J. T. Shen and S. Fan, Phys. Rev. Lett. 95, 213001 (2005).

[10] O. Astafiev, A. M. Zagoskin, A. A. Abdumalikov Jr., Y. A. Pashkin, T. Yamamoto, K. Inomata, Y. Nakamura, and J. S. Tsai, Science 327, 840 (2010).

[11] A. A. Abdumalikov Jr., O. Astafiev, A. M. Zagoskin, Yu. A. Pashkin, Y. Nakamura, and J. S. Tsai, Phys. Rev. Lett. 104, 193601 (2010).

[12] O. Astafiev, Yu. A. Pashkin, Y. Nakamura, J. S. Tsai, A. A. Abdumalikov Jr., and A. M. Zagoskin, Phys. Rev. Lett. 104, 183603 (2010). 
[13] Yu-xi Liu, J. Q. You, L. F. Wei, C. P. Sun, and F. Nori, Phys. Rev. Lett. 95, 087001 (2005).

[14] L. F. Wei, J. R. Johansson, L. X. Cen, S. Ashhab, and Franco Nori, Phys. Rev. Lett. 100, 113601 (2008); W. Z. Jia and L. F. Wei, Phys. Rev. A 82, 013808 (2010).

[15] With the experimental parameters in Ref. [12], numerical calculation shows that if $\left|\Omega_{21}\right|=\Gamma_{21} / 5,\left|\Omega_{31}\right| \geqslant \Gamma_{32},\left|\Omega_{32}\right| \geqslant \Gamma_{32}$, then $\left|\left(\Omega_{21} B\right) /\left(\Gamma_{32} \Omega_{31} \Omega_{32}^{*} / A\right)\right|<0.02,\left|\left(\Omega_{21} B\right) /\left(\Omega_{21} / \Gamma_{21}\right)\right|<$ 0.015 .
[16] M. Baur, S. Filipp, R. Bianchetti, J. M. Fink, M. Goppl, L. Steffen, P. J. Leek, A. Blais, and A. Wallraff, Phys. Rev. Lett. 102, 243602 (2009).

[17] M. A. Sillanpaa, Jian Li, K. Cicak, F. Altomare, J. I. Park, R. W. Simmonds, G. S. Paraoanu, and P. J. Hakonen, Phys. Rev. Lett. 103, 193601 (2009).

[18] W. R. Kelly, Z. Dutton, J. Schlafer, B. Mookerji, T. A. Ohki, J. S. Kline, and D. P. Pappas, Phys. Rev. Lett. 104, 163601 (2010). 